\title{
NEW POSSIBILITIES OPENED BY SYNCHROTRON SPECTROMICROSCOPY IN NEUROBIOLOGY
}

\author{
G. De Stasio \\ Institut de Physique Appliquée, Ecole Polytechnique Fédérale \\ 1015 Lausanne, Switzerland \\ and \\ Istituto di Struttura della Materia del CNR \\ Via Enrico Fermi 38, 00044 Frascati, Roma, Italy
}

\begin{abstract}
The use of synchrotron spectromicroscopy in neurobiology is definitely beyond the feasibility-test stage. It produced a large variety of results, mostly concerning the microscopic localization of transition elements in neural cells. We will first describe the experimental technique, then we will discuss some specific experimental examples, including the uptake of cobalt and zinc in rat cerebellar neurons.
\end{abstract}

PACS numbers: $87.64 . \mathrm{Lg}, 87.80 .+\mathrm{s}, 87.22 .-\mathrm{q}$

\section{Imaging photoelectron spectromicroscopy}

The basic principle of imaging photoelectron spectromicroscopy can be easily understood interpreting it as a type of electron microscopy, in which the source of electrons, instead of being an electron gun, is the sample itself. The sample surface, in fact, when illuminated by X-rays, is a subject to the photoelectric effect, and emits electrons.

The electrons emitted by the sample carry information on the atoms which emitted them. More specifically, on the corresponding chemical elements and on their chemical status, i.e., on the chemical bonds that involve them. This approach to chemical analysis has very extensively been used for decades under the general name of "photoelectron spectroscopy".

Considering the high analytical power of photoelectron spectroscopy, one might wonder why this technique was not combined with electron microscopy to perform the analysis on a microscopic scale. The reason was, until quite recently, that no suitable photon sources existed to provide sufficient photon flux and brightness. This situation, however, has radically changed with the advent of sophisticated synchrotron light sources.

The microscopic-scale version of photoelectron spectroscopy, known as photaelectron spectromicroscopy, was born in the late 1980's [1, 2]. In a few years, 
it has emerged as one of the most powerful experimental techniques for materials science $[1,2]$. Furthermore, the achievement of spatial resolution beyond the typical size of cells has opened the door to the use of spectromicroscopy in biological and medical research [3-5].

Two different approaches to synchrotron spectromicroscopy are possible: imaging and scanning. In the scanning approach, one focuses the photon beam on a microscopic area of the sample, and collects the photoelectrons emitted from this small spot. The sample is mounted on a scanning stage, micrographs of the sample surface can, therefore, be acquired "pixel-by-pixel". Photoemission spectra, as a function of the photoelectron kinetic energy, can be taken from each "pixel" by means of a cylindrical mirror analyzer [2].

The second approach, i.e., imaging spectromicroscopy does not require strong focusing of the photon beam on the sample. In this technique an electron optics system processes the photoelectrons accelerating and magnifying them. It produces microimages whose contrast reflects the differences in the photoelectron emission intensity from point to point on the sample surface. Therefore, "white" in the image corresponds to areas with strong photoelectron emission, and "black" to areas with little or no emission [1].

With imaging spectromicroscopy, detailed spectral information can be extracted from selected small areas of each image, detecting the emitted photoelectron intensity from each micro-area as a function of the photon energy, typically in the photon energy region of an absorption edge of one of the component elements. These plots reflect the local X-ray absorption coefficient, which changes with the chemical state of the element and with the nearest-neighbor coordination around its atoms [6].

Distribution maps of each element can also be obtained by imaging spectromicroscopy. This is obtained simply acquiring one image above an absorption threshold of a specific element, and a second image of the same area below the absorption threshold. The digital subtraction of the two images will reflect the microscopic distribution of that element. For instance, to detect the distribution map of aluminum, we choose the $\mathrm{Al} L_{2,3}$ threshold at $73 \mathrm{eV}$. We acquire one image at $78 \mathrm{eV}$ photon energy in which all the aluminum in the sample is photoemitting, and a second image of exactly the same area at a photon energy of $72 \mathrm{eV}$, in which aluminum is not photoemitting. The only difference between the two identical images, therefore, is the "aluminum brightness". The digital subtraction of the second image from the first one, therefore, reflects the aluminum micro-distribution map [7].

Imaging techniques offer several advantages for biological applications, when compared to scanning approaches. First of all, they produce all points of an image simultaneously, thus the image taking time is much reduced with respect to scanning techniques; this is quite important in biology experiments that require surveying large specimen areas. Second, methods based on changing the photon energy are quick and powerful in performing a microchemical analysis over large areas. Note that changing the photon energy is difficult or impossible for scanning techniques: commonly used "lenses" for photon focusing are photon energy bandpass devices, incompatible to wide-band tuning of the photon energy. 
The specific experiments we will discuss include data taken with the imaging spectromicroscopes X-ray secondary electron-emission microscope (XSEM) and photoelectron emission microscope by synchrotron undulator illumination (microscope à émission de photoélectrons par illumination synchrotronique de type onduleur - MEPHISTO).

The XSEM, developed by Brian Tonner and his co-workers at the University of Wisconsin, was the first instrument in imaging spectromicroscopy [1]. MEPHISTO, developed by our group, is its natural evolution towards higher lateral resolution and better correction of the aberrations in the image produced by the electron optical system.

\section{MEPHISTO}

\section{Microscope à Emission de PHotoélectrons par Illumination Synchrotronique de Type Onduleur}
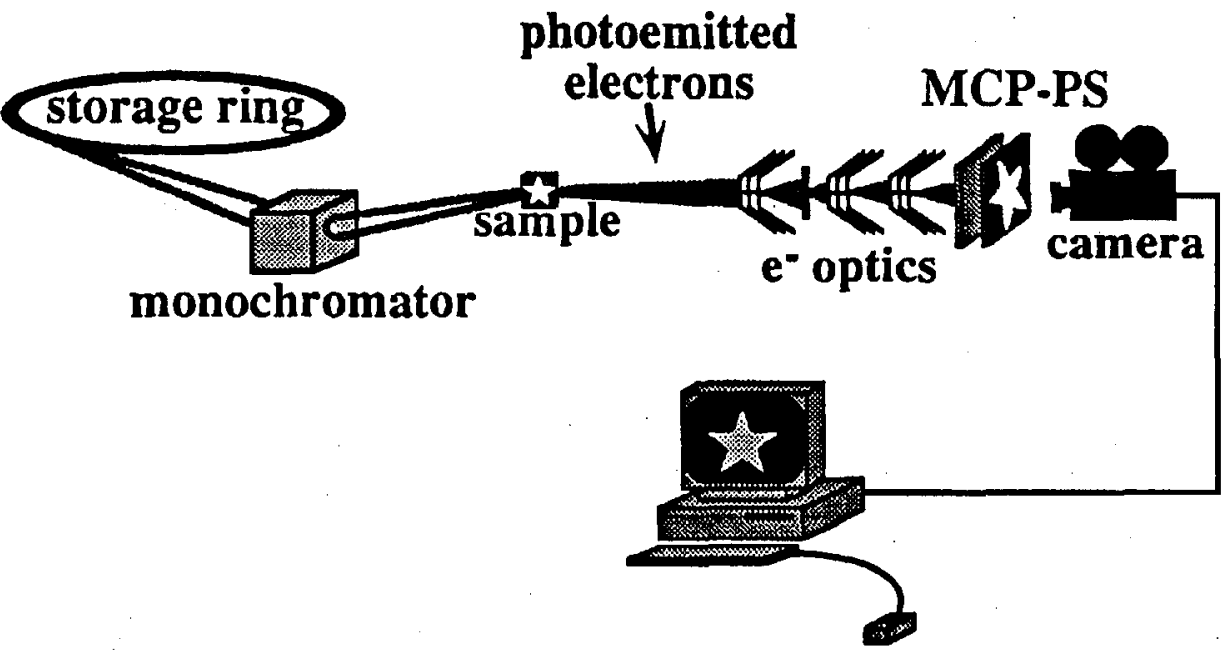

Fig. 1. Artist's view of the MEPHISTO system.

Figure 1 shows an artist's view of the system. The basic philosophy is still quite similar to that of the XSEM or photo-electron emission microscope (PEEM). In all these instruments, a photon beam reaches the sample which is negatively biased with respect to ground. Under X-ray illumination, the sample emits electrons which are accelerated (because of the negative sample bias) towards the system of electrostatic lenses which constitute the electron optics.

Each lens magnifies the photoelectron image; the electron optics thus produces a magnified photoelectron image which reflects the microchemical and geometrical features of the sample. This electron image is then intensified by a microchannel plate device, then converted to a visible image by a phosphorous screen. The video acquisition system is used to monitor the visible image in real time and to take and store digitalized black-and-white individual pictures. 
Systems prior to MEPHISTO could achieve maximum lateral resolutions of the order of 0.5 micron, and partial correction for the image aberrations. Significant improvements have been introduced for MEPHISTO to go beyond these performance limitations.

Specifically, the number of optical elements has been increased and the maximum accelerating voltage has also been augmented. The design lateral resolution is better than any other instrument of the same class operating at the present time. The higher acceleration voltage also improves the instrument performances as far as chromatic aberrations are concerned.

The development of MEPHISTO began in 1995. The construction of the first version of the instrument (with a preliminary electron optics system) initiated on October 18 of the same year and the first images were delivered on November 20. Although not yet reaching the final design performances, this first version produced a large volume of real data. Quite recently, the implementation of the final optics system was completed, and its commissioning stage initiated.

\section{Spectromicroscopy in neurobiology}

The uptake of cobalt by brain cell cultures is extensively investigated because of its importance in understanding several $\mathrm{Co}^{2+}$ related physiological mechanisms such as neuron membrane polarization $[8,9]$. Cobalt accumulation is well known to occur in granule cells in the presence of excitatory amino acids such as kainate $[8,9]$. We obtained with the XSEM data of Fig. 2 evidence of cobalt in granule cells in the absence of kainate [10]. The cells were exposed to a $5 \mathrm{mM}$ solution of $\mathrm{CoCl}_{2}$ in buffer, in the absence of excitatory amino acids, and then carefully washed to remove all the non-uptaken cobalt. In Fig. 2 we present X-ray absorption spectra taken in the spectral region of the Co $L_{2,3}$ edge in different microscopic areas (approximate size: $10 \times 10$ micron $^{2}$ ) of the granule cell culture. The spectra were taken in the correspondingly labeled areas of the photoelectron micrograph of the same figure, and they clearly show the presence of $\mathrm{Co}$, even though no excitatory amino acid was used. This is precisely the result that our present data demonstrate. We find cobalt where other histochemical techniques failed to detect it, probably due to a higher sensitivity of our spectromicroscopic approach to this problem.

Among all the elements studied in neurobiological systems, we present here zinc [11]. The surface sensitivity of spectromicroscopy is usually considered as a limit of this technique. Imaging spectromicroscopy, in fact, probes only the outermost $100-200 \AA$ of the specimen's surface, detecting mostly secondary electrons with low kinetic energy. In the study of the micro-chemical distribution of zinc, this surface sensitivity resulted as an advantage, because it allowed us to discriminate zinc uptake in the "surface" (the membrane) of cells, and their "bulk" (the cytoplasm).

The uptake of zinc by cerebellar rat cultures upon exposure to $\mathrm{ZnCl}_{2}$ solutions was comparatively investigated using two well-known condensed matter physics techniques: imaging spectromicroscopy with the XSEM and inductively-coupled-plasma atomic emission spectroscopy (ICP-AES) [12]. The ICP-AES results clearly demonstrate that the exposure significantly enhances the "bulk" Zn 


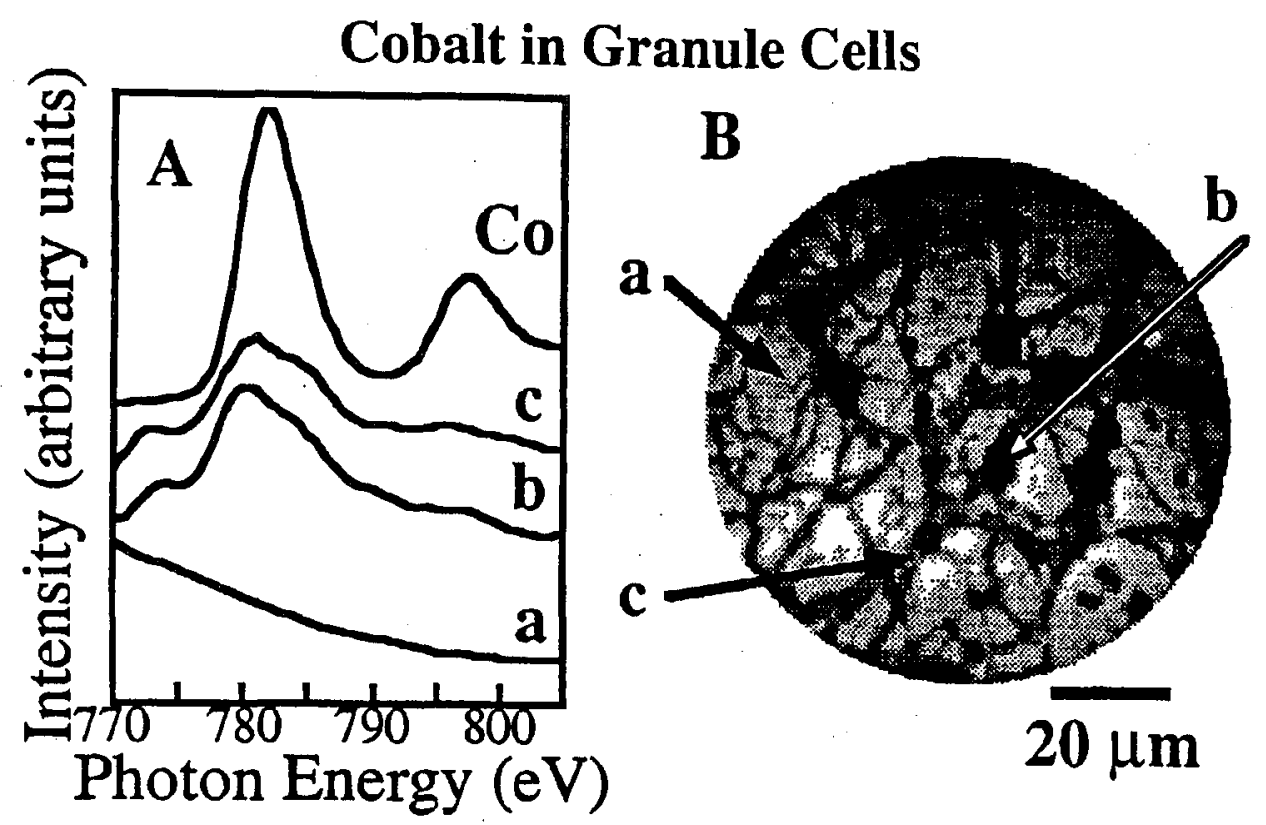

Fig. 2. (A) X-ray absorption spectra taken with imaging photoelectron spectromicroscopy in the two $10 \times 10 \mu \mathrm{m}^{2}$ areas marked as (a), (b) and (c) in micrograph shown in (B), in the photon energy spectral range of the Co $L_{2,3} \mathrm{X}$-ray absorption edge. The top curve is the spectrum of a dried $\mathrm{CoCl}_{2}$ droplet, curve $a$, being from a substrate region does not show any Co-related signal. (B) Synchrotron photoelectron micrograph of a portion of a granule cell culture. The labels (a), (b) and (c) refer to the microscopic areas where the corresponding spectra of (A) were acquired.

concentration with respect to the physiological level, whereas the effect on the "surface" (cell membrane) is negligible.

We observed zinc in a variety of different cell types, including glial cells granule and Purkinje neurons and oligodendrocytes, all exposed to zinc solutions for variable periods of time, and variable exposure solution zinc concentrations. Figure 3 shows an example of the spectromicroscopy results obtained on oligodendrocytes.

We did not observe significant differences in the zinc content between the different cells, different times of exposure or concentrations. We therefore studied the physiologically present zinc, and the absorption spectra demonstrated that the amount of zinc present with or without exposure to a zinc solution was basically comparable.

Parallel experiments performed with ICP-AES, on the other hand, clearly demonstrated a time dependence and a concentration dependence in the zinc uptake increase after the exposure of cell cultures to zinc solutions.

We concluded that the exposure does not significantly affect the zinc concentration in the region probed by spectromicroscopy - the "surface" - but it does 


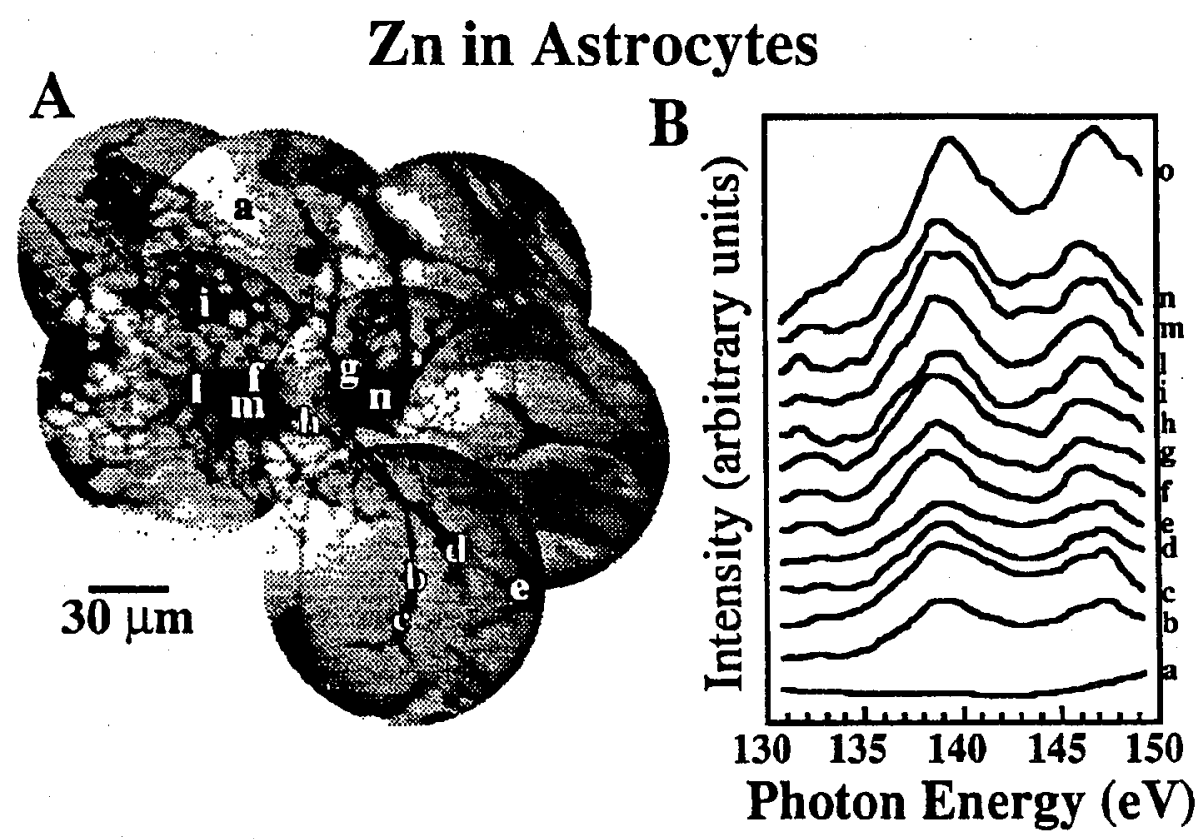

Fig. 3. (A) Combination of spectromicroscopy micrographs of astrocytes, found as a subpopulation of a culture with prevailing glial cells, after exposure to a $5 \mathrm{mM} \mathrm{ZnCl} 2$ solution for $20 \mathrm{~min}$. The photon energy was $130 \mathrm{eV}$. The letters identify the microscopic $\left(\approx 3 \times 3\right.$ micron $^{2}$ ) regions in which the spectra of Fig. $3 B$ were taken. (B) XSEM (X-ray absorption) spectra from these regions. The data were background-corrected, normalized and twice-smoothed over five-point sets. Curve $a$ refers to a substrate area and is the only one not showing the typical $\mathrm{Zn} 3 \mathrm{~s}$ absorption threshold line shape. Curve $o$ is a reference spectrum from a dried droplet of a zinc sulfate solution.

increase the overall zinc concentration (ICP data). Thus, the effect of exposure is primarily a "bulk" phenomenon.

The main difference between spectromicroscopy and ICP-AES, in fact, is surface sensitivity. In imaging spectromicroscopy, one probes the first 100-200 $\AA$ of the sample surface. In the case of cells, therefore, the probed depth includes the cell membrane ( $50 \AA$ thick), part of the membrane proteins and only a small portion of the cytoplasm.

On the other hand, ICP-AES is not surface sensitive at all, it measures the concentration of elements in solution, after suspending and solubilizing the entire cells in nitric acid. The most plausible explanation of the differences between our spectromicroscopy and ICP-AES data, is, therefore, the fact that the former investigates the "surface" of our specimens whereas the latter investigates their "bulk", where "surface" and "bulk" in the present case mean the cell's membrane and its cytoplasm. Our data indicate that $\mathrm{Zn}$ is physiologically present, independent of any exposure, both in the membrane and in the cytoplasm. This is not surprising, for example in light of the existence of $\mathrm{Zn}$ binding proteins. 
The differences between spectromicroscopy and ICP-AES data, on the other hand, indicate that the artificial $\mathrm{Zn}$ exposures significantly increase only the $\mathrm{Zn}$ content in the cytoplasm. This could be either due to a large physiological amount of $\mathrm{Zn}$ in the membrane, which is only marginally affected by the exposures, or to a more effective mechanism for exposure-caused uptake in the cytoplasm or else to a combination of both effects. Our results cannot definitely rule out anyone of these effects. Speculatively, we tend to favor the first conjecture, because of the hypothesized involvement of $\mathrm{Zn}$ in synaptic transmission [13].

\section{Acknowledgments}

We are indebted to Brian P. Tonner, Delio Mercanti, M. Teresa Ciotti, Giorgio Margaritondo, Paolo Perfetti and Mario Capozi for their participation to the experiments. Research supported by the Fonds National Suisse de la Recherche Scientifique, the Ecole Polytechnique Fédérale de Lausanne, the Italian Consiglio Nazionale delle Ricerche. All the spectromicroscopy experiments were performed at the University of Wisconsin, Synchrotron Radiation Center, a facility funded by the US National Science Foundation.

\section{References}

[1] B.P. Tonner, D. Dunham, Nucl. Instrum. Methods A 347, 436 (1994) and the references therein; B.P. Tonner, G.R. Harp, S.F. Koranda, J. Zhang, Rev. Sci. Instrum. 63, 564 (1992).

[2] F. Cerrina, A.K. Ray-Chaudhuri, W. Ng, S. Liang, S. Singh, J.T. Welnak, J.P. Wallace, C. Capasso, J.H. Underwood, J.B. Kortright, R.C.C. Perera, G. Margaritondo, Appl. Phys. Lett. 63, 63 (1993).

[3] G. De Stasio, P. Perfetti, W. Ng, A.K. Ray-Chaudhuri, S. Liang, S. Singh, R.K. Cole, Z.Y. Guo, J. Wallace, C. Capasso, F. Cerrina, D. Mercanti, M.T. Ciotti, F. Gozzo, G. Margaritondo, Phys. Rev. E 48, 1478 (1993).

[4] G. De Stasio, D. Mercanti, M.T. Ciotti, D. Dunham, T.C. Droubay, B.P. Tonner, P. Perfetti, G. Margaritondo, NeuroReport 5, 1973 (1994).

[5] G. De Stasio, D. Dunham, B.P. Tonner, D. Mercanti, M.T. Ciotti, P. Perfetti, G. Margaritondo, J. Synch. Rad. 2, 106 (1995) and the references therein.

[6] W. Gudat, C. Kunz, Phys. Rev. Lett. 29, 169 (1972).

[7] G. De Stasio, D. Dunham, B.P. Tonner, D. Mercanti, M.T. Ciotti, C. Coluzza, P. Perfetti, G. Margaritondo, NeuroReport 4, 1175 (1993).

[8] R.M. Pruss, R.L. Akeson, M.M. Racke, J.L. Wilburn, Neuron 7, 509 (1991).

[9] S.J. Butke, H. Yin, J.H. Weiss, NeuroReport 6, $6 \check{2} 9$ (1995).

[10] G.F. Lorusso, G. De Stasio, D. Mercanti, M.T. Ciotti, D. Perret, A. Merbach, P. Perfetti, G. Margaritondo, in preparation.

[11] G. De Stasio, S. Pochon, G.F. Lorusso, B.P. Tonner, D. Mercanti, M.T. Ciotti, N. Oddo, P. Galli, P. Perfetti, G. Margaritondo, J. Phys. D 29, 2209 (1996).

[12] A. Varma, Hanbook of Inductively Coupled Plasma Atomic Emission Spectroscopy, CRC, Boca Raton 1991, p. 380.

[13] J. Koh, D.W. Choi, J. Neurosci. 8, 2164 (1988). 